\title{
Advances in the Molecular Catalysis of Dioxygen Reduction
}

\section{Supporting Information}

\author{
Charles W. Machan \\ machan@virginia.edu \\ University of Virginia \\ McCormick Road PO Box 400319 \\ Charlottesville, VA 22904-4319 \\ ORCID 0000-0002-5182-1138
}

\section{Contents}

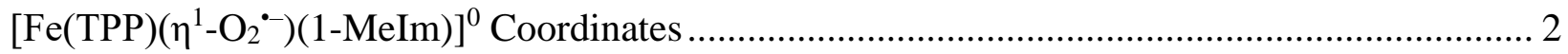

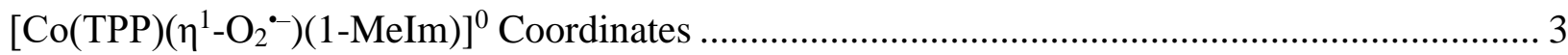

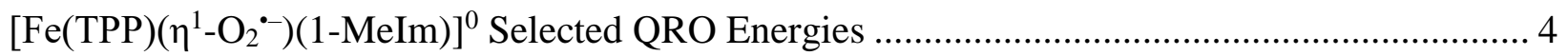

$\left[\mathrm{Co}(\mathrm{TPP})\left(\eta^{1}-\mathrm{O}_{2}{ }^{-}\right)(1-\mathrm{MeIm})\right]^{0}$ Selected QRO Energies........................................................... 5

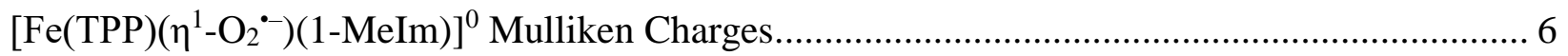

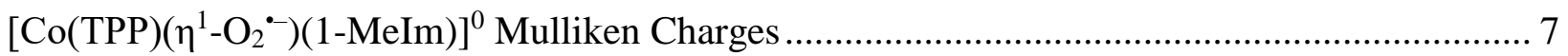

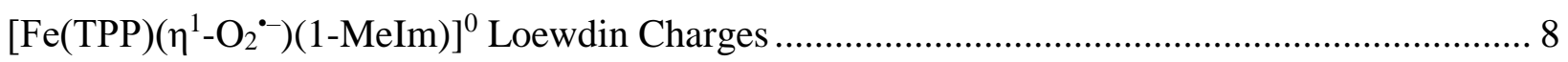

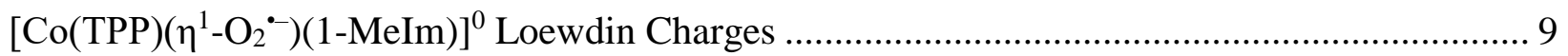


$\left[\mathrm{Fe}(\mathrm{TPP})\left(\eta^{1}-\mathrm{O}_{2}{ }^{--}\right)(1-\mathrm{MeIm})\right]^{0}$ Coordinates $\mathrm{S}=0 ;-3590.726461640822$ Hartree

$\begin{array}{lrrr}\mathrm{Fe} & 2.432576 & 16.115618 & 8.003586 \\ \mathrm{~N} & 2.023277 & 15.126773 & 6.286911 \\ \mathrm{~N} & 0.460579 & 16.074748 & 8.437037 \\ \mathrm{~N} & 2.817073 & 17.031421 & 9.738551 \\ \mathrm{~N} & 4.387651 & 16.064774 & 7.600622 \\ \mathrm{O} & 2.219955 & 17.627945 & 7.143769 \\ \mathrm{O} & 3.005585 & 18.607738 & 7.291553 \\ \mathrm{C} & 2.952187 & 14.620056 & 5.413195 \\ \mathrm{C} & 0.793181 & 14.950038 & 5.700604 \\ \mathrm{C} & -0.569240 & 15.711439 & 7.606142 \\ \mathrm{C} & -0.103415 & 16.384552 & 9.649997 \\ \mathrm{C} & 1.920158 & 17.269140 & 10.752223 \\ \mathrm{C} & 4.014061 & 17.578945 & 10.137663 \\ \mathrm{C} & 5.389892 & 16.689687 & 8.301739 \\ \mathrm{C} & 4.995179 & 15.356357 & 6.591514 \\ \mathrm{C} & 2.288471 & 14.115727 & 4.228317 \\ \mathrm{H} & 2.789086 & 13.673972 & 3.371021 \\ \mathrm{C} & 0.953981 & 14.343763 & 4.395195 \\ \mathrm{H} & 0.147070 & 14.117675 & 3.704360 \\ \mathrm{C} & -1.828068 & 15.789326 & 8.319108 \\ \mathrm{H} & -2.800218 & 15.546837 & 7.898554 \\ \mathrm{C} & -1.537351 & 16.190341 & 9.590183 \\ \mathrm{H} & -2.226507 & 16.359658 & 10.413230 \\ \mathrm{C} & 2.572006 & 17.990754 & 11.825171 \\ \mathrm{H} & 2.097065 & 18.300010 & 12.751769 \\ \mathrm{C} & 3.857804 & 18.215023 & 11.428087 \\ \mathrm{H} & 4.640312 & 18.740932 & 11.967214 \\ \mathrm{C} & 6.675024 & 16.350221 & 7.730795 \\ \mathrm{H} & 7.635343 & 16.695303 & 8.103410 \\ \mathrm{C} & 6.432763 & 15.504444 & 6.687817 \\ \mathrm{H} & 7.157754 & 15.028703 & 6.033421 \\ \mathrm{C} & 4.346395 & 14.653136 & 5.566685 \\ \mathrm{C} & -0.445194 & 15.241453 & 6.290296 \\ \mathrm{C} & 0.563437 & 16.917720 & 10.760512 \\ \mathrm{C} & 5.235580 & 17.457577 & 9.464124 \\ \mathrm{C} & 5.173161 & 13.965630 & 4.529961 \\ \mathrm{C} & 5.979935 & 14.698455 & 3.642899 \\ \mathrm{H} & 6.007052 & 15.787993 & 3.720302 \\ \mathrm{C} & 6.731782 & 14.048239 & 2.660456 \\ \mathrm{H} & 7.348986 & 14.633973 & 1.974420 \\ \mathrm{C} & 6.688411 & 12.654172 & 2.550161 \\ \mathrm{H} & 7.274572 & 12.145177 & 1.780915 \\ \mathrm{C} & 5.887046 & 11.915056 & 3.426803 \\ \mathrm{H} & 5.846822 & 10.825275 & 3.347144\end{array}$

$\begin{array}{llll}\mathrm{C} & 5.134966 & 12.566463 & 4.408203 \\ \mathrm{H} & 4.509450 & 11.988082 & 5.092031 \\ \mathrm{C} & -1.694988 & 15.001607 & 5.511279 \\ \mathrm{C} & -2.521990 & 16.083588 & 5.163275 \\ \mathrm{H} & -2.234051 & 17.093677 & 5.463449 \\ \mathrm{C} & -3.697270 & 15.878314 & 4.436004 \\ \mathrm{H} & -4.326292 & 16.731258 & 4.170150 \\ \mathrm{C} & -4.065886 & 14.586197 & 4.047568 \\ \mathrm{H} & -4.985746 & 14.424159 & 3.480808 \\ \mathrm{C} & -3.253062 & 13.501481 & 4.392782 \\ \mathrm{H} & -3.537300 & 12.488322 & 4.098501 \\ \mathrm{C} & -2.077464 & 13.707572 & 5.119999 \\ \mathrm{H} & -1.451177 & 12.856819 & 5.397292 \\ \mathrm{C} & -0.239637 & 17.184301 & 11.992974 \\ \mathrm{C} & -0.591201 & 18.493124 & 12.360552 \\ \mathrm{H} & -0.268369 & 19.328965 & 11.736024 \\ \mathrm{C} & -1.354192 & 18.729160 & 13.507795 \\ \mathrm{H} & -1.623229 & 19.753106 & 13.778466 \\ \mathrm{C} & -1.777321 & 17.659021 & 14.303022 \\ \mathrm{H} & -2.371954 & 17.844682 & 15.200681 \\ \mathrm{C} & -1.434980 & 16.351448 & 13.943479 \\ \mathrm{H} & -1.760818 & 15.510381 & 14.559963 \\ \mathrm{C} & -0.672331 & 16.116469 & 12.795718 \\ \mathrm{H} & -0.404379 & 15.094870 & 12.515610 \\ \mathrm{C} & 6.446062 & 18.122960 & 10.029844 \\ \mathrm{C} & 7.064528 & 19.170928 & 9.326733 \\ \mathrm{H} & 6.642899 & 19.494800 & 8.372890 \\ \mathrm{C} & 8.202883 & 19.798962 & 9.839415 \\ \mathrm{H} & 8.668788 & 20.615171 & 9.281835 \\ \mathrm{C} & 8.741538 & 19.386835 & 11.062760 \\ \mathrm{H} & 9.632079 & 19.876293 & 11.463629 \\ \mathrm{C} & 8.137049 & 18.340822 & 11.768204 \\ \mathrm{H} & 8.556013 & 18.007381 & 12.720295 \\ \mathrm{H} & 6.998368 & 17.713944 & 11.255208 \\ \mathrm{H} & 6.535082 & 16.890443 & 11.803048 \\ \mathrm{~N} & 2.614450 & 14.341313 & 8.933378 \\ \mathrm{~N} & 2.216962 & 12.272464 & 9.569121 \\ \mathrm{C} & 3.576139 & 13.983694 & 9.852734 \\ \mathrm{H} & 4.361960 & 14.668965 & 10.156215 \\ \mathrm{C} & 3.339530 & 12.692911 & 10.258333 \\ \mathrm{H} & 3.856162 & 12.045279 & 10.961976 \\ & 1.812532 & 13.294511 & 8.782368 \\ \mathrm{H} & 1.578311 & 10.969845 & 9.684875 \\ \mathrm{H} & 2.303697 & 10.174857 & 9.463287 \\ \mathrm{H} & 10.915174 & 8.967920 \\ \mathrm{H} & & & \\ \mathrm{H} & & \\ \mathrm{H} & & \\ \mathrm{H} & & \\ \mathrm{H} & & \end{array}$


$\left[\mathrm{Co}(\mathrm{TPP})\left(\eta^{1}-\mathrm{O}_{2}{ }^{-}\right)(1-\mathrm{MeIm})\right]^{0}$ Coordinates

$\mathrm{S}=1 / 2 ;-3709.799976414137$ Hartree

$\begin{array}{lrrc}\mathrm{Co} & 2.423297 & 16.074969 & 8.033506 \\ \mathrm{~N} & 2.023020 & 15.158174 & 6.287571 \\ \mathrm{~N} & 0.456886 & 16.098201 & 8.452399 \\ \mathrm{~N} & 2.804971 & 17.054878 & 9.747363 \\ \mathrm{~N} & 4.380454 & 16.088492 & 7.587101 \\ \mathrm{C} & 2.947074 & 14.654560 & 5.406598 \\ \mathrm{C} & 0.788507 & 14.984663 & 5.706657 \\ \mathrm{C} & -0.572022 & 15.730744 & 7.620919 \\ \mathrm{C} & -0.108225 & 16.410645 & 9.664397 \\ \mathrm{C} & 1.912356 & 17.299664 & 10.762518 \\ \mathrm{C} & 4.005736 & 17.594185 & 10.140134 \\ \mathrm{C} & 5.380524 & 16.699611 & 8.300861 \\ \mathrm{C} & 4.990956 & 15.376079 & 6.582659 \\ \mathrm{C} & 2.277490 & 14.162862 & 4.220856 \\ \mathrm{H} & 2.774254 & 13.728748 & 3.357409 \\ \mathrm{C} & 0.943908 & 14.390758 & 4.395641 \\ \mathrm{H} & 0.133740 & 14.172096 & 3.706259 \\ \mathrm{C} & -1.830778 & 15.811436 & 8.332491 \\ \mathrm{H} & -2.802835 & 15.570013 & 7.911309 \\ \mathrm{C} & -1.541646 & 16.218106 & 9.602210 \\ \mathrm{H} & -2.231932 & 16.392913 & 10.423123 \\ \mathrm{C} & 2.569939 & 18.023021 & 11.830434 \\ \mathrm{H} & 2.098710 & 18.338077 & 12.756889 \\ \mathrm{C} & 3.855839 & 18.237167 & 11.429114 \\ \mathrm{H} & 4.643001 & 18.761116 & 11.963328 \\ \mathrm{C} & 6.668362 & 16.353381 & 7.736764 \\ \mathrm{H} & 7.628136 & 16.690483 & 8.117878 \\ \mathrm{C} & 6.428625 & 15.514182 & 6.688705 \\ \mathrm{H} & 7.154675 & 15.035377 & 6.037747 \\ \mathrm{C} & 4.341637 & 14.675583 & 5.557519 \\ \mathrm{C} & -0.448575 & 15.263131 & 6.304394 \\ \mathrm{C} & 0.556369 & 16.947418 & 10.774632 \\ \mathrm{C} & 5.226568 & 17.465215 & 9.465243 \\ \mathrm{C} & 5.161880 & 13.971131 & 4.527978 \\ \mathrm{C} & 5.992184 & 14.683244 & 3.645716 \\ \mathrm{H} & 6.042488 & 15.772103 & 3.719771 \\ \mathrm{C} & 6.737805 & 14.013327 & 2.671714 \\ \mathrm{H} & 7.373239 & 14.583263 & 1.989035 \\ \mathrm{C} & 6.665136 & 12.620206 & 2.565109 \\ \mathrm{H} & 7.246572 & 12.096232 & 1.802382 \\ \mathrm{C} & 5.840525 & 11.901491 & 3.437274 \\ \mathrm{H} & 5.777326 & 10.812572 & 3.360767 \\ \mathrm{C} & 5.094411 & 12.572434 & 4.409832 \\ \mathrm{H} & 4.451389 & 12.009908 & 5.090675\end{array}$

\begin{tabular}{|c|c|c|c|}
\hline $\mathrm{C}$ & -1.698652 & 15.002835 & 5.531913 \\
\hline $\mathrm{C}$ & -2.541679 & 16.069719 & 5.176763 \\
\hline $\mathrm{H}$ & -2.267017 & 17.086514 & 5.466544 \\
\hline C & -3.716272 & 15.840849 & 4.455166 \\
\hline $\mathrm{H}$ & -4.358178 & 16.682102 & 4.183183 \\
\hline C & -4.067594 & 14.539986 & 4.079936 \\
\hline $\mathrm{H}$ & -4.986678 & 14.359439 & 3.517543 \\
\hline $\mathrm{C}$ & -3.238184 & 13.470196 & 4.432092 \\
\hline $\mathrm{H}$ & -3.508387 & 12.450443 & 4.147423 \\
\hline $\mathrm{C}$ & -2.063429 & 13.699883 & 5.153471 \\
\hline $\mathrm{H}$ & -1.423264 & 12.861059 & 5.435304 \\
\hline C & -0.245953 & 17.213257 & 12.007072 \\
\hline C & -0.585904 & 18.522810 & 12.383053 \\
\hline $\mathrm{H}$ & -0.254632 & 19.359889 & 11.764688 \\
\hline $\mathrm{C}$ & -1.348592 & 18.757797 & 13.530745 \\
\hline $\mathrm{H}$ & -1.608825 & 19.782249 & 13.807952 \\
\hline $\mathrm{C}$ & -1.782782 & 17.686157 & 14.317974 \\
\hline $\mathrm{H}$ & -2.377141 & 17.871161 & 15.215954 \\
\hline $\mathrm{C}$ & -1.452018 & 16.377929 & 13.949992 \\
\hline $\mathrm{H}$ & -1.786541 & 15.5 & 14.560193 \\
\hline $\mathrm{C}$ & -0.689889 & 16.143834 & 12.801745 \\
\hline $\mathrm{H}$ & -0.430953 & 15.121764 & 12.515049 \\
\hline $\mathrm{C}$ & 6.442589 & 18.116518 & 10.036413 \\
\hline $\mathrm{C}$ & 7.075262 & 19.159190 & 9.338139 \\
\hline $\mathrm{H}$ & 6.659188 & 19.492177 & 8.385034 \\
\hline $\mathrm{C}$ & 8.221972 & 19.769593 & 9.853427 \\
\hline $\mathrm{H}$ & 8.699130 & 20.581482 & 9.299087 \\
\hline $\mathrm{C}$ & 8.755094 & 19.344855 & 11.074863 \\
\hline $\mathrm{H}$ & 9.652655 & 19.819883 & 11.477323 \\
\hline $\mathrm{C}$ & 8.136094 & 18.304490 & 11.776029 \\
\hline $\mathrm{H}$ & 8.550462 & 17.961352 & 12.726668 \\
\hline $\mathrm{C}$ & 6.989015 & 17.695340 & 11.260363 \\
\hline $\mathrm{H}$ & 6.514971 & 16.875396 & 11.804248 \\
\hline $\mathrm{N}$ & 2.631101 & 14.236222 & 8.998998 \\
\hline $\mathrm{N}$ & 2.277940 & 12.144255 & 9.582193 \\
\hline $\mathrm{C}$ & 3.545353 & 13.904845 & 9.970655 \\
\hline $\mathrm{H}$ & 4.280640 & 14.618980 & 10.331578 \\
\hline $\mathrm{C}$ & 3.336438 & 12.597456 & 10.345376 \\
\hline $\mathrm{H}$ & 3.833058 & 11.959556 & 11.072159 \\
\hline $\mathrm{C}$ & 1.883815 & 13.164911 & 8.783114 \\
\hline $\mathrm{C}$ & 1.697269 & 10.810737 & 9.626029 \\
\hline $\mathrm{H}$ & 1.323813 & 10.593472 & 10.636706 \\
\hline $\mathrm{H}$ & 2.450740 & 10.059436 & 9.350233 \\
\hline $\mathrm{H}$ & 0.862881 & 10.763342 & 8.915474 \\
\hline $\mathrm{H}$ & 1.064448 & 13.089765 & 8.071347 \\
\hline $\mathrm{O}$ & 2.156301 & 17.895881 & 7.014709 \\
\hline $\mathrm{O}$ & 2.978848 & 18.809806 & 7.235760 \\
\hline
\end{tabular}


$\left[\mathrm{Fe}(\mathrm{TPP})\left(\eta^{1}-\mathrm{O}_{2}{ }^{--}\right)(1-\mathrm{MeIm})\right]^{0}$ Selected QRO Energies

\begin{tabular}{lll} 
Orbital (occ) & \multicolumn{1}{c}{ a.u. } & \multicolumn{1}{c}{$\mathbf{e V}$} \\
$190(2)$ & -0.260392 & -7.086 \\
$191(2)$ & -0.259693 & -7.067 \\
$192(2)$ & -0.256517 & -6.98 \\
$193(2)$ & -0.253988 & -6.911 \\
$194(2)$ & -0.253686 & -6.903 \\
$195(2)$ & -0.252983 & -6.884 \\
$196(2)$ & -0.2509 & -6.827 \\
$197(2)$ & -0.244245 & -6.646 \\
$198(2)$ & -0.234158 & -6.372 \\
$199(2)$ & -0.227394 & -6.188 \\
$200(2)$ & -0.22397 & -6.095 \\
$201(2)$ & -0.203025 & -5.525 \\
$202(2)$ & -0.190541 & -5.185 \\
$203(0)$ & -0.126821 & -3.451
\end{tabular}


$\left[\mathrm{Co}(\mathrm{TPP})\left(\eta^{1}-\mathrm{O}_{2}{ }^{--}\right)(1-\mathrm{MeIm})\right]^{0}$ Selected QRO Energies

$\begin{array}{lcc}\text { Orbital (occ) } & \mathbf{a . u .} & \mathbf{e V} \\ 190(2) & -0.260951 & -7.101 \\ 191(2) & -0.260496 & -7.088 \\ 192(2) & -0.259194 & -7.053 \\ 193(2) & -0.256563 & -6.981 \\ 194(2) & -0.253788 & -6.906 \\ 195(2) & -0.253734 & -6.904 \\ 196(2) & -0.252935 & -6.883 \\ 197(2) & -0.243366 & -6.622 \\ 198(2) & -0.237956 & -6.475 \\ 199(2) & -0.237403 & -6.46 \\ 200(2) & -0.203392 & -5.535 \\ 201(2) & -0.198591 & -5.404 \\ 202(2) & -0.181469 & -4.938 \\ 203(1) & -0.166335 & -4.526 \\ 204(0) & -0.090665 & -2.467\end{array}$


$\left[\mathrm{Fe}(\mathrm{TPP})\left(\eta^{1}-\mathrm{O}_{2}{ }^{--}\right)(1-\mathrm{MeIm})\right]^{0}$ Mulliken Charges

* - Fe-bound $\mathrm{O}$ atom

$44 \mathrm{C}: \quad 0.010897$

$45 \mathrm{H}:-0.006458$

MULLIKEN ATOMIC CHARGES

$46 \mathrm{C}:-0.054772$

$47 \mathrm{C}: \quad 0.004404$

$48 \mathrm{H}:-0.004678$

0 Fe: 0.519046

$49 \mathrm{C}:-0.002278$

$1 \mathrm{~N}:-\mathbf{0 . 2 2 2 9 2 3}$

$50 \mathrm{H}: \quad 0.006263$

$2 \mathrm{~N}:-\mathbf{0 . 2 2 6 0 6 2}$

$51 \mathrm{C}: 0.003848$

$52 \mathrm{H}: 0.006555$

$53 \mathrm{C}: 0.002247$

$54 \mathrm{H}: \quad 0.002020$

$55 \mathrm{C}:-0.000586$

$56 \mathrm{H}:-0.003507$

$57 \mathrm{C}:-0.067009$

$58 \mathrm{C}: 0.012876$

$59 \mathrm{H}:-0.005225$

$60 \mathrm{C}:-0.000458$

$61 \mathrm{H}: 0.002609$

$62 \mathrm{C}: 0.002738$

$63 \mathrm{H}: 0.006333$

$64 \mathrm{C}: 0.000930$

$65 \mathrm{H}: 0.005451$

66 C : 0.008756

$67 \mathrm{H}:-0.007693$

$68 \mathrm{C}:-0.073465$

$69 \mathrm{C}: \quad 0.016197$

$70 \mathrm{H}:-0.008690$

$71 \mathrm{C}:-0.007603$

$72 \mathrm{H}: \quad 0.005623$

$73 \mathrm{C}: \quad 0.010715$

$74 \mathrm{H}: \quad 0.004512$

$75 \mathrm{C}:-0.000312$

$76 \mathrm{H}: \quad 0.002371$

$77 \mathrm{C}: \quad 0.003924$

$78 \mathrm{H}:-0.002268$

$79 \mathrm{~N}:-0.206765$

$80 \mathrm{~N}:-0.194292$

$81 \mathrm{C}: 0.060733$

$82 \mathrm{H}: 0.031143$

$83 \mathrm{C}: 0.044781$

$84 \mathrm{H}: \quad 0.043050$

$85 \mathrm{C}: 0.168341$

86 C : 0.128923

$87 \mathrm{H}: \quad 0.060247$

$88 \mathrm{H}: \quad 0.060172$

$89 \mathrm{H}: 0.059938$

$90 \mathrm{H}: 0.061001$ 
$\left[\mathrm{Co}(\mathrm{TPP})\left(\eta^{1}-\mathrm{O}_{2}{ }^{-}\right)(1-\mathrm{MeIm})\right]^{0}$ Mulliken Charges

* - Co-bound $\mathrm{O}$ atom

$44 \mathrm{C}:-0.054453$

$45 \mathrm{C}: 0.004763$

MULLIKEN ATOMIC CHARGES

$46 \mathrm{H}:-0.004763$

$47 \mathrm{C}:-0.002026$

$48 \mathrm{H}: \quad 0.006545$

0 Co: 0.545629

$49 \mathrm{C}: 0.004587$

$50 \mathrm{H}: \quad 0.006241$

$51 \mathrm{C}: 0.002650$

$52 \mathrm{H}: 0.002310$

$53 \mathrm{C}:-0.001680$

$54 \mathrm{H}:-0.003563$

$55 \mathrm{C}:-0.067852$

$56 \mathrm{C}: 0.013504$

$57 \mathrm{H}:-0.005735$

$58 \mathrm{C}:-0.000659$

$59 \mathrm{H}: \quad 0.002552$

$60 \mathrm{C}: 0.002987$

$61 \mathrm{H}: \quad 0.006344$

$62 \mathrm{C}: 0.001397$

$63 \mathrm{H}: 0.005377$

$64 \mathrm{C}: \quad 0.009267$

$65 \mathrm{H}:-0.007140$

$66 \mathrm{C}:-0.071582$

$67 \mathrm{C}: \quad 0.017088$

$68 \mathrm{H}:-0.009754$

$69 \mathrm{C}:-0.009060$

$70 \mathrm{H}: \quad 0.005897$

$71 \mathrm{C}: \quad 0.011879$

$72 \mathrm{H}: 0.004215$

$73 \mathrm{C}: 0.000901$

$74 \mathrm{H}: \quad 0.002068$

$75 \mathrm{C}: \quad 0.002929$

$76 \mathrm{H}:-0.001957$

$77 \mathrm{~N}$ : -0.201210

$78 \mathrm{~N}:-0.196688$

$79 \mathrm{C}: \quad 0.045057$

$80 \mathrm{H}: \quad 0.028540$

$81 \mathrm{C}: 0.041887$

$82 \mathrm{H}: 0.040806$

$83 \mathrm{C}: 0.151746$

$84 \mathrm{C}: 0.126806$

$85 \mathrm{H}: 0.058365$

$86 \mathrm{H}: 0.059798$

$87 \mathrm{H}: \quad 0.058847$

$88 \mathrm{H}: 0.059271$

*89 0 : $\quad-\mathbf{0 . 1 2 0 8 1 3}$

900 : $-\mathbf{- 0 . 1 4 2 4 3 3}$ 
$\left[\mathrm{Fe}(\mathrm{TPP})\left(\eta^{1}-\mathrm{O}_{2}{ }^{--}\right)(1-\mathrm{MeIm})\right]^{0}$ Loewdin Charges

* - Fe-bound $\mathrm{O}$ atom

\section{LOEWDIN ATOMIC CHARGES}

\begin{tabular}{|c|c|}
\hline & \\
\hline $\begin{array}{l}\text { 0 Fe: } \\
1 \mathrm{~N}:\end{array}$ & $\begin{array}{r}\mathbf{0 . 0 7 9 5 9 0} \\
-0.058594\end{array}$ \\
\hline $2 \mathrm{~N}:$ & -0.058201 \\
\hline $3 \mathrm{~N}:$ & -0.051872 \\
\hline $4 \mathrm{~N}:$ & -0.051280 \\
\hline *50 : & 0.038611 \\
\hline $60:$ & -0.215609 \\
\hline $7 \mathrm{C}:$ & 0.006959 \\
\hline $8 \mathrm{C}:$ & 0.003631 \\
\hline $9 \mathrm{C}:$ & 0.002880 \\
\hline 10 C: & 0.007480 \\
\hline $11 \mathrm{C}:$ & 0.008478 \\
\hline $12 \mathrm{C}:$ & 0.011712 \\
\hline $13 \mathrm{C}:$ & 0.011121 \\
\hline $14 \mathrm{C}:$ & 0.008316 \\
\hline $15 \mathrm{C}:$ & -0.041276 \\
\hline $16 \mathrm{H}:$ & 0.032995 \\
\hline $17 \mathrm{C}:$ & -0.041789 \\
\hline $18 \mathrm{H}:$ & 0.033222 \\
\hline 19 C : & -0.041084 \\
\hline $20 \mathrm{H}:$ & 0.033084 \\
\hline $21 \mathrm{C}:$ & -0.041896 \\
\hline $22 \mathrm{H}:$ & 0.033299 \\
\hline $23 \mathrm{C}:$ & -0.037821 \\
\hline $24 \mathrm{H}:$ & 0.032983 \\
\hline $25 \mathrm{C}:$ & -0.040594 \\
\hline $26 \mathrm{H}:$ & 0.033353 \\
\hline $27 \mathrm{C}:$ & -0.040174 \\
\hline $28 \mathrm{H}:$ & 0.033353 \\
\hline $29 \mathrm{C}:$ & -0.038168 \\
\hline $30 \mathrm{H}:$ & 0.033391 \\
\hline $31 \mathrm{C}:$ & -0.033575 \\
\hline $32 \mathrm{C}$ : & -0.036878 \\
\hline $33 \mathrm{C}$ : & -0.033311 \\
\hline $34 \mathrm{C}$ : & -0.030920 \\
\hline $35 \mathrm{C}:$ & -0.019561 \\
\hline $36 \mathrm{C}$ : & -0.022461 \\
\hline $37 \mathrm{H}:$ & 0.034077 \\
\hline $38 \mathrm{C}:$ & -0.030528 \\
\hline $39 \mathrm{H}:$ & 0.036185 \\
\hline $40 \mathrm{C}:$ & -0.030415 \\
\hline $41 \mathrm{H}:$ & 0.035083 \\
\hline $42 \mathrm{C}:$ & -0.032111 \\
\hline
\end{tabular}

$43 \mathrm{H}: \quad 0.036734$

$44 \mathrm{C}:-0.020910$

$45 \mathrm{H}: 0.033403$

$46 \mathrm{C}:-0.019473$

$47 \mathrm{C}:-0.021105$

$48 \mathrm{H}: \quad 0.033894$

49 C : -0.029528

$50 \mathrm{H}: \quad 0.036110$

$51 \mathrm{C}:-0.030340$

$52 \mathrm{H}: \quad 0.035125$

53 C : -0.029166

$54 \mathrm{H}: \quad 0.035289$

$55 \mathrm{C}:-0.023220$

$56 \mathrm{H}: \quad 0.033069$

$57 \mathrm{C}:-0.018475$

$58 \mathrm{C}:-0.022818$

$59 \mathrm{H}: \quad 0.033590$

$60 \mathrm{C}:-0.028930$

$61 \mathrm{H}: \quad 0.035288$

62 C : -0.031482

$63 \mathrm{H}: \quad 0.034970$

$64 \mathrm{C}:-0.029030$

$65 \mathrm{H}: \quad 0.035822$

66 C : -0.023341

$67 \mathrm{H}: \quad 0.033049$

68 C : -0.020407

69 C : -0.019668

$70 \mathrm{H}: \quad 0.033312$

$71 \mathrm{C}:-0.030204$

$72 \mathrm{H}: \quad 0.035751$

73 C : -0.029887

$74 \mathrm{H}: \quad 0.034683$

$75 \mathrm{C}:-0.029185$

$76 \mathrm{H}: \quad 0.035394$

$77 \mathrm{C}:-0.022823$

$78 \mathrm{H}: 0.033088$

$79 \mathrm{~N}:-0.020870$

$80 \mathrm{~N}: \quad 0.068048$

81 C : -0.016945

$82 \mathrm{H}: 0.032065$

83 C : -0.017820

$84 \mathrm{H}: 0.042136$

85 C : 0.061924

86 C : 0.053261

$87 \mathrm{H}: \quad 0.042257$

$88 \mathrm{H}: \quad 0.042743$

$89 \mathrm{H}: \quad 0.045867$

$90 \mathrm{H}: \quad 0.037072$ 
$\left[\mathrm{Co}(\mathrm{TPP})\left(\eta^{1}-\mathrm{O}_{2}{ }^{--}\right)(1-\mathrm{MeIm})\right]^{0}$ Loewdin Charges

* - Co-bound $\mathrm{O}$ atom

$43 \mathrm{H}: \quad 0.033406$

$44 \mathrm{C}:-0.019502$

$45 \mathrm{C}:-0.021120$

LOEWDIN ATOMIC CHARGES

$46 \mathrm{H}: \quad 0.033943$

$47 \mathrm{C}:-0.029473$

$48 \mathrm{H}: \quad 0.036248$

0 Co: 0.178753

49 C : -0.029986

$50 \mathrm{H}: \quad 0.035144$

$51 \mathrm{C}:-0.029128$

$52 \mathrm{H}: \quad 0.035346$

$53 \mathrm{C}:-0.022876$

$54 \mathrm{H}: \quad 0.033137$

$55 \mathrm{C}:-0.018814$

$56 \mathrm{C}:-0.022451$

$57 \mathrm{H}: \quad 0.033581$

$58 \mathrm{C}:-0.028856$

$59 \mathrm{H}: \quad 0.035249$

$60 \mathrm{C}:-0.031268$

$61 \mathrm{H}: 0.035010$

$62 \mathrm{C}:-0.028895$

$63 \mathrm{H}: 0.035890$

$64 \mathrm{C}:-0.022996$

$65 \mathrm{H}: 0.033132$

66 C : -0.020496

67 C : -0.019554

$68 \mathrm{H}: 0.032982$

$69 \mathrm{C}:-0.030397$

$70 \mathrm{H}: \quad 0.035800$

$71 \mathrm{C}:-0.029626$

$72 \mathrm{H}: \quad 0.034649$

$73 \mathrm{C}:-0.029040$

$74 \mathrm{H}: \quad 0.035338$

$75 \mathrm{C}:-0.022726$

$76 \mathrm{H}: 0.033196$

$77 \mathrm{~N} ：-0.029818$

$78 \mathrm{~N}: \quad 0.064335$

79 C : -0.025636

$80 \mathrm{H}: \quad 0.030653$

81 C: -0.021782

$82 \mathrm{H}: \quad 0.040653$

83 C : 0.049826

84 C : 0.051818

$85 \mathrm{H}: 0.041639$

$86 \mathrm{H}: \quad 0.041791$

$87 \mathrm{H}: \quad 0.045328$

$88 \mathrm{H}: \quad 0.035617$

*89 O : $\quad-\mathbf{0 . 0 5 8 5 4 5}$

90 O : $-\mathbf{- 0 . 1 5 8 0 2 5}$ 